\title{
Aeolian Tone from a Semi-Circular Cylinder in a Stream*
}

\author{
Takayuki Yamagata1, Nozomi Saito², Nobuyuki Fujisawa1 \\ ${ }^{1}$ Visualization Research Center, Niigata University, Niigata, Japan \\ ${ }^{2}$ Graduate School of Science and Technology, Niigata University, Niigata, Japan \\ Email: yamagata@eng.niigata-u.ac.jp
}

Received 14 November 2015; accepted 9 January 2016; published 29 January 2016

Copyright () 2016 by authors and Scientific Research Publishing Inc.

This work is licensed under the Creative Commons Attribution International License (CC BY). http://creativecommons.org/licenses/by/4.0/

C. (7) Open Access

\begin{abstract}
Aeolian tone from a semi-circular cylinder in a uniform flow is studied experimentally for various angles of attack. It is found that the peak sound spectrum of the Aeolian tone from the semi-circular cylinder is smaller than that from the circular cylinder and the lowest sound is observed around the zero angle of attack. This is due to the reduction in the fluctuating lift force on the semi-circular cylinder compared to that of the circular cylinder. This result suggests the validity of the analogy between the sound pressure level and the fluctuating lift force on a semi-circular cylinder in a stream. The flow visualization study also supports these results.
\end{abstract}

\section{Keywords}

Aeolian Tone, Semi-Circular Cylinder, Fluctuating Lift Force, Angle of Attack, Flow Induced Sound

\section{Introduction}

Aeolian tone generated from a bluff body in a stream has been a topic of interest for many years, because it is closely related to the environmental noise problem induced by the stream. Examples of such flow noise can be found in wind turbines, vehicles, cables, buildings, and so on. So far, the flow noise from the bluff body in a stream has been investigated to understand the physics behind the Aeolian tone generated by the fluid structure interaction.

The Aeolian tone from the circular cylinder has been studied by many researchers, which is summarized in review papers [1] [2]. These studies are concerned with the aerodynamic characteristics of the circular cylinder in a uniform flow, which is closely related to the generation mechanism of Aeolian tone. The experimental results on the unsteady fluid forces of the circular cylinder indicate that the fluctuating lift coefficient is dependent on the Reynolds number of the flow and is highly correlated with the Aeolian tone from the circular cylinder [3].

\footnotetext{
*Part of this work was presented at $12^{\text {th }}$ International Conference on Fluid Dynamics, October 2015, Sendai.
} 
Since then, the experimental and numerical studies on the unsteady flow field and the aerodynamic sound from the circular cylinder have been carried out to explore the mechanism of the sound generation from the circular cylinder in a uniform flow [4]-[12]. It should be mentioned that the sound sources of the Aeolian tone are known to be located around the separation points and along the separating shear layer, which are measured by particle image velocimetry combined with microphone technique [12].

Compared to the studies on the aerodynamic characteristics of the circular cylinder, there are fewer studies on the rectangular cylinder [13] [14] and the semi-circular cylinder [15]. Furthermore, there are much fewer studies on the aeroacoustics than those of aerodynamics of these cylinders. Fujita [16] reported the characteristics of the Aeolian tone for various configurations of two-dimensional cylinders and showed that the sound pressure level of the Aeolian tone from a square cylinder is reduced by the introduction of rounded corners on the front side, which is similar in shape to the semi-circular cylinder. As far as the authors know, there are no previous studies on the Aeolian tone from a semi-circular cylinder in a stream, while this configuration is often encountered in engineering applications, such as the wing mirror of automobiles, the Savonius wind turbines [17], and the heat exchangers and so on.

In this study, the Aeolian tone from the semi-circular cylinder in a uniform flow is investigated experimentally for various angles of attack by measuring the sound spectrum, fluctuating lift coefficient and flow visualization by smoke-wire method.

\section{Experimental Methods}

Figure 1 shows an experimental setup for the measurement of Aeolian tone and the aerodynamic characteristics of the semi-circular cylinder in a uniform flow. The measurements were carried out in a wind tunnel, which has a cross-section of $190 \mathrm{~mm} \times 190 \mathrm{~mm}$. The diameter of the semi-circular cylinder is $D=15 \mathrm{~mm}$, so that the aspect ratio of the cylinder is 12.7 . Note that the blockage of the wind tunnel is less than $8 \%$ and the influence of the blockage on the fluid forces is the same order of magnitude to the uncertainty of fluid force measurement, so that the correction of the blockage effect was not applied to the present result. Mean velocity of the experiment was set at $U=30 \mathrm{~m} / \mathrm{s}$, which corresponds to the Reynolds number $\operatorname{Re}(=U D / v)=3 \times 10^{4}$, where $v$ is kinematic viscosity of air. Note that the independence of the Reynolds number on this result was confirmed in the Reynolds number range $R e=(1.9-3.3) \times 10^{4}$. The turbulence intensity of the wind tunnel is $1 \%$ of the free-stream velocity.

Aeolian tone generated from the semi-circular cylinder was measured by a microphone having a diameter of $12.7 \mathrm{~mm}$. The microphone was located above the cylinder with a distance of $130 \mathrm{~mm}$ as shown in Figure 1. The top wall of the wind tunnel is made of urethane foam to minimize the flow noise, while the other walls are made of acrylic resin for flow visualization purposes. The microphone has a flat frequency response in a range of 20 $\mathrm{Hz}$ to $8 \mathrm{kHz}$. The output signals from the microphone are recorded digitally to a computer via the analog to digital converter and the data are stored on the memory.

In order to understand the physics of Aeolian tone variation with the angle of attack, unsteady fluid forces acting on the cylinder were measured by using four strain gauges attached to one side of the square rod. The cylinder was fixed on both ends outside the wind tunnel as shown in Figure 1. The strain gauges are pasted on each flat surface of the square rod. The sensing section of the fluid force is the central $90 \mathrm{~mm}$ of the cylinder to avoid the influence of the boundary layer developing on the side walls of the wind tunnel. Figure 2 shows the configuration of the strain gauges on the square rod. Two strain gauges on the opposite sides of the square rod
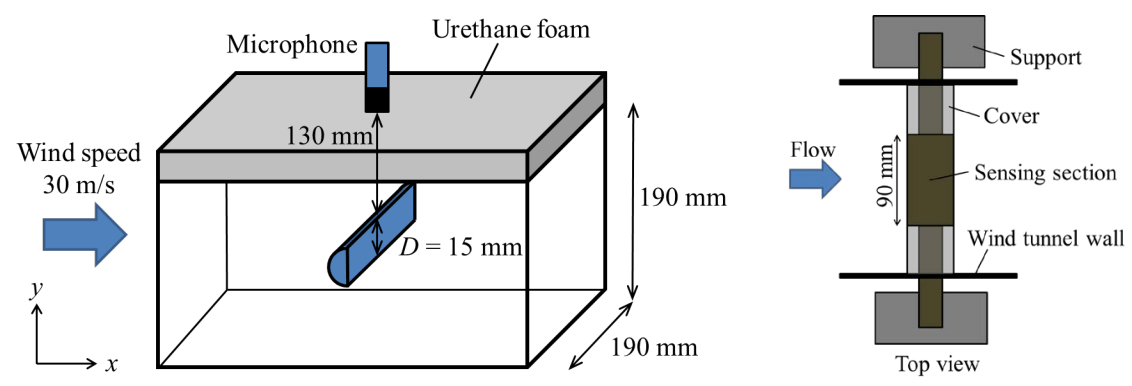

Figure 1. Experimental setup. 


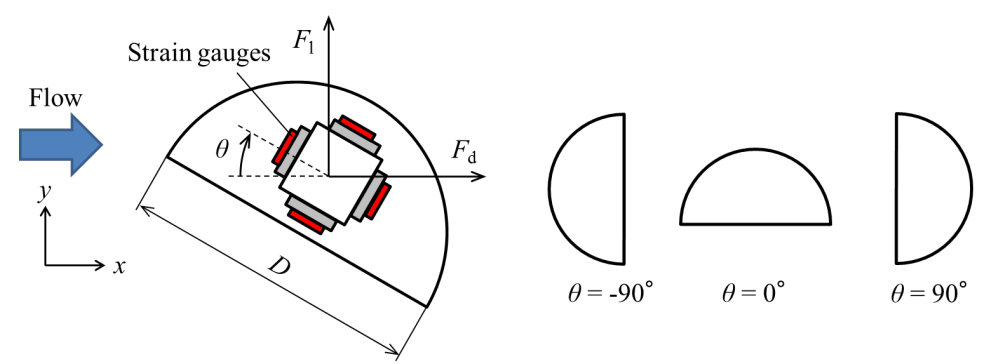

Figure 2. Semi-circular cylinder and strain gauges.

compose a half bridge of two active gauge method. The two sets of the half bridge allow the measurement of the strains in orthogonal directions. The maximum frequency response of the strain gauges is $2.5 \mathrm{kHz}$, which is much higher than the frequency of the noise. The drag and lift forces are evaluated from the strain measurements, where the drag and lift coefficients are defined by

$$
\begin{aligned}
& C_{d}=F / \frac{1}{2} \rho U^{2} D L \\
& C_{l}=F_{l} / \frac{1}{2} \rho U^{2} D L
\end{aligned}
$$

( $D$ : diameter of the cylinder, $F_{\mathrm{d}}, F_{\mathrm{l}}$ : drag and lift forces, respectively, $L$ : length of sensing section, $\rho$ : density of air). The relation between the strain and the fluid forces was calibrated for each angle of attack by a static loading test before the experiment, because the strain gauges are fixed on the cylinder. The uncertainty of the fluid force measurement was evaluated as $7.7 \%$, where the calibration error $7 \%$ is the major error source. In order to evaluate the influence of the angle of attack $\theta$ (Figure 2) on the aerodynamic characteristics, the semi-circular cylinder is rotated around the gravity center. The sampling rate of the signal acquisition was $10 \mathrm{kHz}$ and the total sampling time was $60 \mathrm{~s}$. The sound spectra were evaluated by Fast Fourier Transform (FFT) analysis of the recorded data.

In order to confirm the change of flow pattern, flow fields around the semi-circular cylinder were visualized by a smoke-wire method. A nichrome wire of diameter $0.1 \mathrm{~mm}$ was installed just behind the cylinder. Liquid paraffin was painted on the wire by using a brush before electric heating of the wire. The mean velocity of the wind tunnel was set to $U=3 \mathrm{~m} / \mathrm{s}$ to obtain a clear smoke image, which corresponds to the Reynolds number $R e$ $=3 \times 10^{3}$. The visualization images were recorded at $1000 \mathrm{frame} / \mathrm{s}$ using a high-speed camera having an image resolution of $1016 \times 1016$ pixels.

\section{Results and Discussion}

\subsection{Sound Spectrum and Strouhal Number}

Figure 3 shows an example of sound spectra for some angles of attack of a semi-circular cylinder $(\theta=0,-90$, 90 degrees) at $R e=3 \times 10^{4}$, which is compared with that of the circular cylinder at the same Reynolds number. The background noise is $20 \mathrm{~dB}$ smaller than the Aeolian tone. The sound spectra of the semi-circular cylinder show peaks at vortex shedding frequency, while the peak frequency changes with the angle of attack. It should be mentioned that the peak frequency of the circular cylinder is $370 \mathrm{~Hz}$, which corresponds to the Strouhal number $S t(=f D / U)=0.19$ in agreement with the reported value in literature [3]. The second peak of lower frequency in the sound spectrum of the circular cylinder may be due to the effect of end conditions of the cylinder, which produces the irregular motion of the vortex shedding [18]. It is interesting that the peak magnitude of the sound pressure spectra for the semi-circular cylinder at $\theta=0$ and -90 degrees is much lower than that of the circular cylinder.

Figure 4 shows the variations of peak sound pressure levels of the semi-circular cylinder with the angle of attack $\theta$, which is compared with those of the circular cylinder. It is found that the peak magnitude is lowest around zero angle of attack and it becomes large around $\theta=-30$ degrees and $\theta=45$ degrees or over. It should be mentioned that the peak sound spectra of the semi-circular cylinder is lower than that of the circular cylinder irrespective of the angle of attack. This indicates that the aeroacoustic performance of the semi-circular cylinder is better than that of the circular cylinder. 


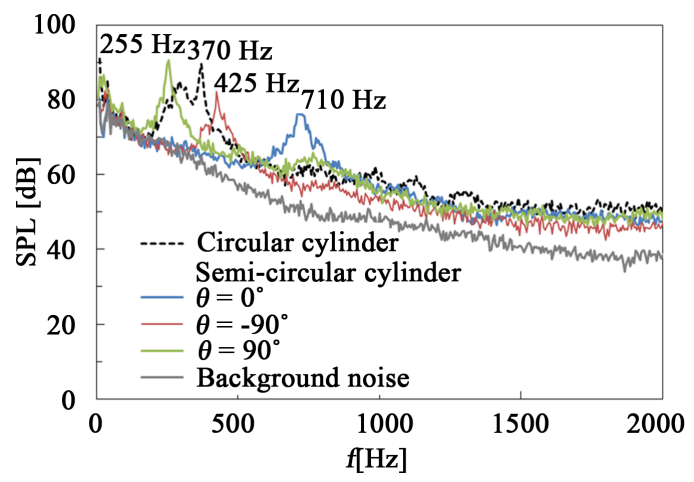

Figure 3. Spectrum of sound pressure level.

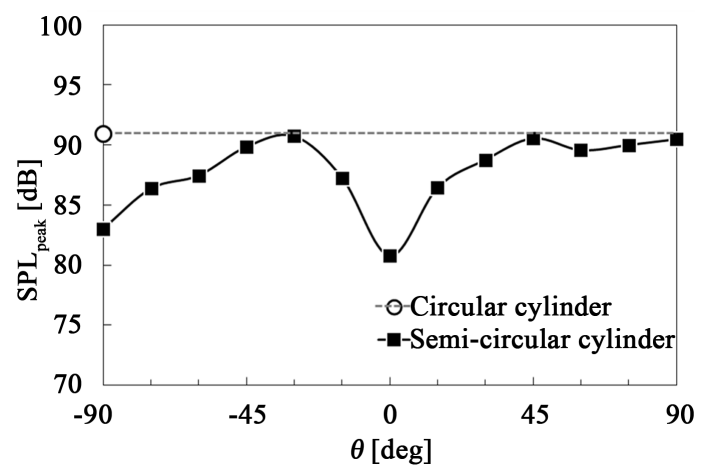

Figure 4. Peak magnitude of sound pressure level.

Figure 5 shows the variations of Strouhal number with the angle of attack $\theta$ for the semi-circular cylinder, which is compared to the case of circular cylinder. The Strouhal number of the semi-circular cylinder shows highest value ( $\mathrm{St}=0.37$ ) around $\theta=15$ degrees and it is lowest $(\mathrm{St}=0.12)$ around $\theta=90$ degrees, which is reflected by the variation of frontal area of the semi-circular cylinder. On the other hand, the Strouhal number stays around 0.2 in the range of angle of attack $\theta=-90$ to -45 degrees and the result agrees with that of the circular cylinder. These results indicate that the variation of the Strouhal number with the angle of attack is caused by the wake-width variation, which will be seen in the flow visualization pictures in Figure 8, while the high Strouhal number at 15 degrees and the low Strouhal number at -15 degrees is due to the change of the separation point along the surface.

\subsection{Fluid Forces on Semi-Circular Cylinder}

Figure 6 shows the drag and lift coefficients of the semi-circular cylinders for various angles of attack, which are compared with those of the semi-circular cylinder by Takizawa et al. [15] at $R e=6.7 \times 10^{4}$. Note that the drag coefficient $C_{\mathrm{d}}=1.0$ of the circular cylinder is the average value over the various angles of attack, which agrees with that in literature [3]. The drag coefficient of the semi-circular cylinder around zero angle of attack is lower than those of the circular cylinder. This is similarly observed in the result of semi-circular cylinder by Takizawa et al. [15], which was evaluated by integration of surface pressure measurement. With increasing the angle of attack to positive direction, the drag coefficient increases and it becomes larger than that of the circular cylinder. With decreasing the angle of attack to negative direction, the drag coefficient increases gradually and it becomes the same level as that of the circular cylinder at $\theta=-90$ degrees. The lift coefficient shows symmetric distribution with respect to $\theta=0$ degrees reflecting the angle of attack variation of the frontal area. Although the negative lift coefficient is found around zero angle of attack, they increase with increasing the magnitude of the angle of attack in both positive and negative directions and they come to zero lift coefficient at $\theta= \pm 90$ degrees due to the axial symmetry of the semi-circular cylinder with respect to the flow direction. It should be mentioned that the deviation of the present result of drag and lift coefficients from that of Takizawa et al. [15] may be due 

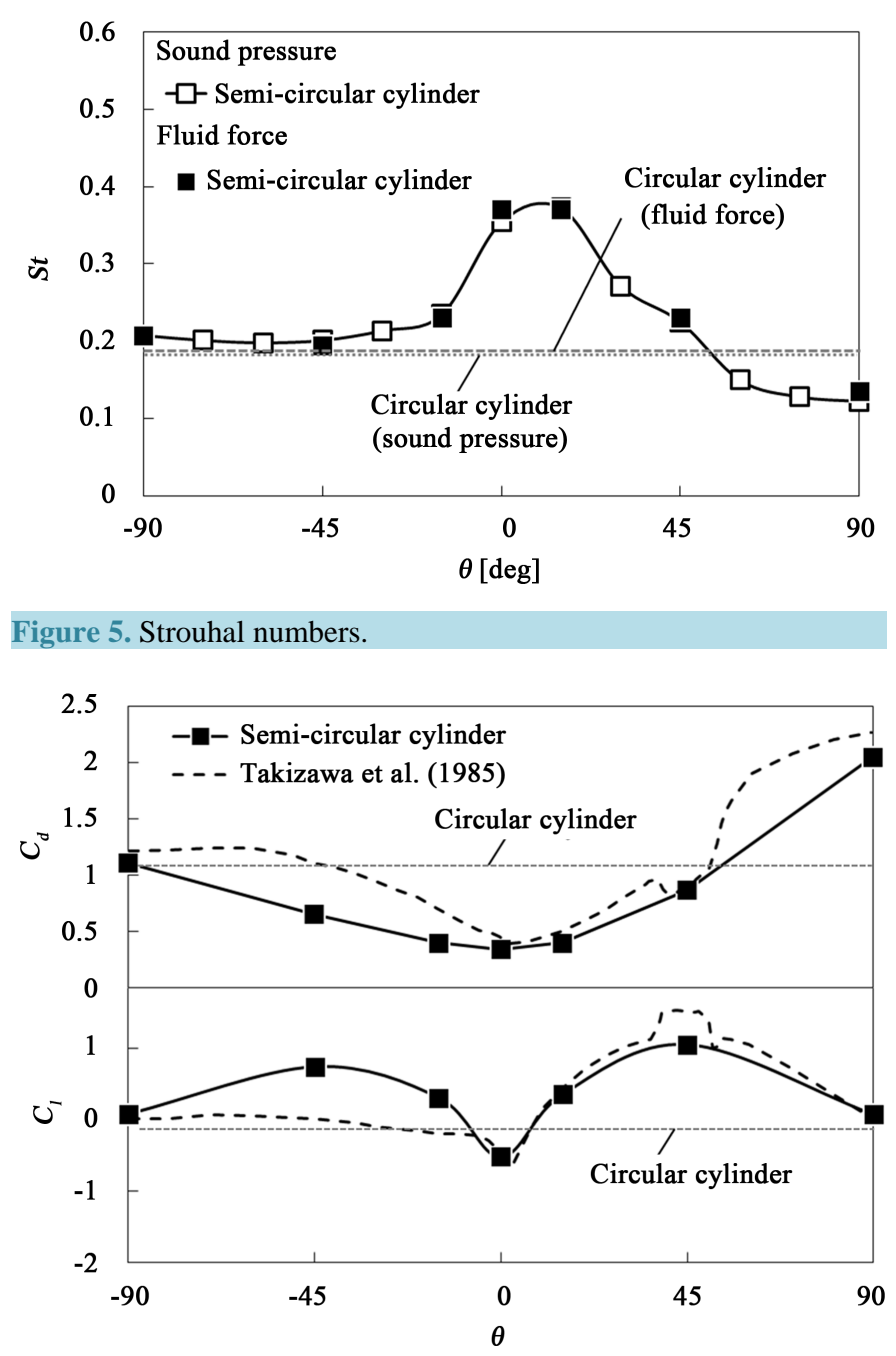

Figure 6. Mean drag and lift coefficients.

to the influence of Reynolds number, which results in larger difference in drag and lift coefficients on the negative $\theta$.

Figure 7 shows the distribution of fluctuating lift coefficient $C_{l r m s}$ of the semi-circular cylinders for various angles of attack, which is compared with that of the circular cylinder. Note that the error bar indicates the data scattering in the experiment. The fluctuating lift coefficient of the semi-circular cylinder is very small around zero angle of attack. It increases gradually with increasing the magnitude of angle of attack to positive and negative directions, while it is much smaller than that of the circular cylinder. This result indicates that the fluctuating lift coefficient can be reduced by using the semi-circular cylinder instead of the circular cylinder and it is applicable to all angles of attack. It should be mentioned that the distribution of the fluctuating lift coefficient is very similar to that of the overall sound pressure level with respect to the angle of attack variation as shown in the same figure. This result indicates that the overall sound pressure level is highly correlated with the fluctuating lift coefficient, which implies that the Curle's theory [19] under the assumption of compact body is applicable to the Aeolian tone generation from a semi-circular cylinder in a stream. However, these distributions does not agree well with each other, which is found at the local peak of SPL around $\theta=-45$ degrees. This discrepancy may be due to the effect of the sound sources in the flow fields and directional characteristics of the Aeolian tones of the semi-circular cylinder [19]. The present result shows that the fluctuating lift coefficient at $\theta=-90$ degree is lower than that at $\theta=90$ degree, which indicates that the flat back structure is more effective than the flat front structure for reducing the fluctuating lift and the peak sound spectrum. 


\subsection{Smoke-Wire Visualization of Flow Field}

Figure 8 shows the smoke-wire flow visualization of flow field around the semi-circular cylinder at $\theta=0$ degree (a), -15 degree (b), 15 degree (c), -90 degree (d), 90 degree (e), respectively, which are compared with the result of the circular cylinder (f). The smoke-wire experiments were conducted at $R e=3 \times 10^{3}$, where the smoke-wire is located at $10 \mathrm{~mm}$ downstream of the cylinder center. These flow visualization pictures show that the flow fields around the semi-circular cylinder are similar to that of the circular cylinder, while the wake width downstream of the semi-circular cylinder are modified by the angle of attack variation, which reflects the variation of the frontal area of the semi-circular cylinder. Note that the wake width, which is presented in Figure 8, is defined by the disturbed width of the smoke on the smoke-wire position downstream of the cylinder. The smallest wake width of $0.8 \mathrm{D}$ is observed around zero angle of attack, while it decreases slightly with increasing the positive angle of attack 15 degrees and the increase is found as $1 \mathrm{D}$ by the negative angle of attack -15 degrees. The wake width further increases to $1.1 \mathrm{D}$ at the negative angle of attack -90 degrees, which is the same order as the circular cylinder. Moreover, it becomes the maximum 1.7 $\mathrm{D}$ at positive angle of attack 90 degrees. These observations of wake flow field are consistent with the angle of attack variation of the Strouhal number, the peak sound spectra and the fluctuating lift forces on the semi-circular cylinder.

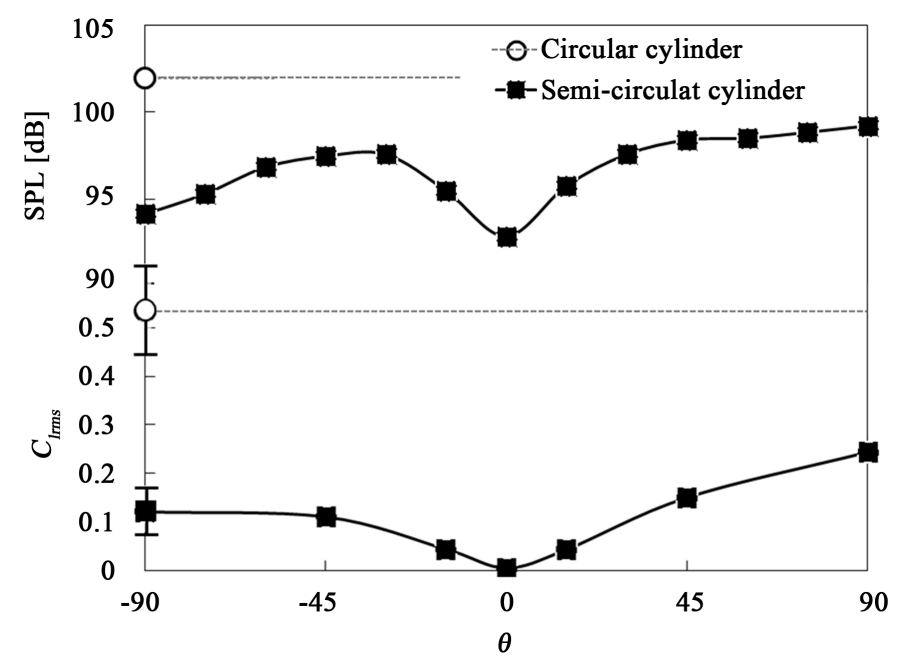

Figure 7. Fluctuating lift coefficient and sound pressure level.

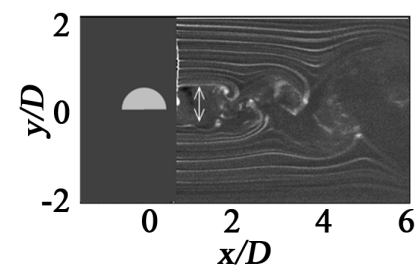

(a)

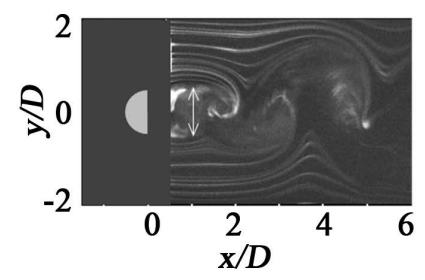

(d)

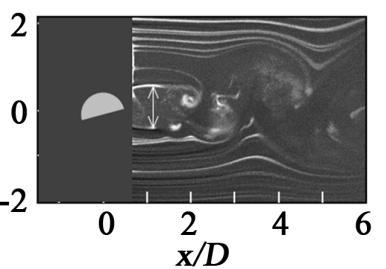

(b)

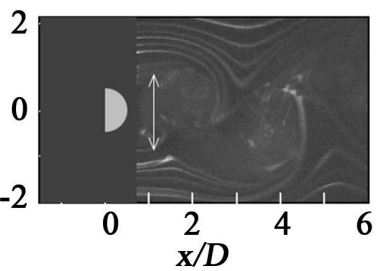

(e)

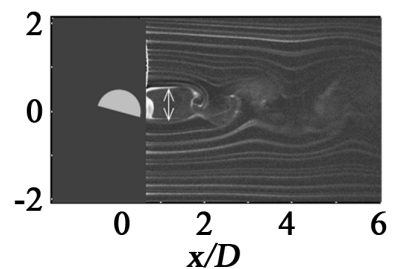

(c)

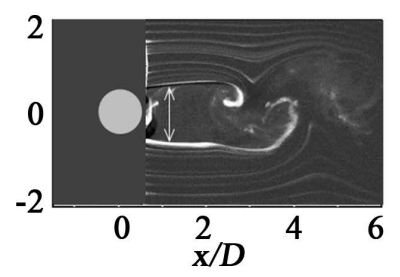

(f)

Figure 8. Smoke-wire flow visualizations of flow around circular and semi-circular cylinders. (a) $\theta=0$ degree; (b) $\theta=-15$ degree; (c) $\theta=15$ degrees; (d) $\theta=-90$ degrees; (e) $\theta=90$ degrees; (f) Circular cylinder. 


\section{Conclusion}

The Aeolian tone from a semi-circular cylinder in a uniform flow was investigated experimentally for various angles of attack by measuring the sound spectrum and the mean and fluctuating fluid forces. The experimental results show that the peak sound spectrum from the semi-circular cylinder is smaller than that of the circular cylinder in the whole angles of attack and the lowest one is observed around zero angle of attack. This is due to the generation of smaller fluctuating lift force on the semi-circular cylinder compared to that of the circular cylinder. The variation of the peak sound spectra with the angle of attack is similarly observed to the variations of fluctuating lift force, which supports the validation of the analogy between the sound pressure level and the fluctuating lift force on the semi-circular cylinder.

\section{Acknowledgements}

The authors would like to express thanks to Dr. T. Nakano (Mitsuba Co. Ltd.), Dr. Y. Oguma (Nihon University) and Mr. S. Kulakit (former graduate student of Niigata University) for their help during the course of this work.

\section{References}

[1] Blevins, R.D. (1984) Review of Sound Induced by Vortex Shedding from Cylinders. Journal of Sound and Vibration, 92, 455-470. http://dx.doi.org/10.1016/0022-460X(84)90191-3

[2] Blake, W.K. (1986) Mechanics of Flow-Induced Sound and Vibration, Vol. 1, General Concepts and Elementary Sources. Academic Press, Cambridge, Massachusetts, 44-64.

[3] Norberg, C. (2003) Fluctuating Lift on a Circular Cylinder: Review and New Measurements. Journal of Fluids and Structures, 17, 57-96. http://dx.doi.org/10.1016/S0889-9746(02)00099-3

[4] Inoue, O. and Hatakeyama, N. (2002) Sound Generation by a Two-Dimensional Circular Cylinder in a Uniform Flow. Journal of Fluid Mechanics, 471, 285-314. http://dx.doi.org/10.1017/S0022112002002124

[5] Fujisawa, N., Tanahashi, S. and Srinivas, K. (2005) Evaluation of Pressure Field and Fluid Forces on a Circular Cylinder with and without Rotational Oscillation Using Velocity Data from PIV Measurement. Measurement Science and Technology, 16, 89-996. http://dx.doi.org/10.1088/0957-0233/16/4/011

[6] Fujisawa, N., Asano, Y., Arakawa, C. and Hashimoto, T. (2005) Computational and Experimental Study on Flow around a Rotationally Oscillating Circular Cylinder in a Uniform Flow. Journal of Wind Engineering and Industrial Aerodynamics, 93, 137-153. http://dx.doi.org/10.1016/j.jweia.2004.11.002

[7] Cheong, C., Joseph, P., Park, Y. and Lee, S. (2008) Computation of Aeolian Tone from a Circular Cylinder Using Source Models. Applied Acoustics, 69, 110-126. http://dx.doi.org/10.1016/j.apacoust.2006.10.004

[8] Rokugou, A., Kiwata, T., Okajima, A., Kimura, S. and Yamamoto, H. (2008) Numerical Analysis of Aerodynamic Sound Radiated from Rectangular Cylinder. Journal of Wind Engineering and Industrial Aerodynamics, 96, 22032216. http://dx.doi.org/10.1016/j.jweia.2008.02.045

[9] Henning, A., Kaepernick, K., Ehrenfried, K., Koop, L. and Dillmann, A. (2008) Investigation of Aeroacoustic Noise Generation by Simultaneous Particle Image Velocimetry and Microphone Measurements. Experiments in Fluids, 45, 1073-1085. http://dx.doi.org/10.1007/s00348-008-0528-y

[10] Kaltenbacher, M., Escobar, M. Becker, S. and Ali, I. (2010) Numerical Simulation of flow-Induced Noise Using LES/SAS and Lighthill's Acoustic Analogy. International Journal for Numerical Methods in Fluids, 63, 1103-1122.

[11] Moore, P., Lorenzoni, V. and Scarano, F. (2011) Two Techniques for PIV-Based Aeroacoustic Prediction and Their Application to a Rod-Airfoil Experiment. Experiments in Fluids, 50, 877-885. http://dx.doi.org/10.1007/s00348-010-0932-y

[12] Oguma, Y., Yamagata, T. and Fujisawa, N. (2013) Measurement of Sound Source Distribution around a Circular Cylinder in a Uniform Flow by Combined Particle Image Velocimetry and Microphone Technique. Journal of Wind Engineering and Industrial Aerodynamics, 118, 1-11. http://dx.doi.org/10.1016/j.jweia.2013.04.003

[13] Okajima, A. (1990) Numerical Simulation of Flow around Rectangular Cylinders. Journal of Wind Engineering and Industrial Aerodynamics, 33, 171-180. http://dx.doi.org/10.1016/0167-6105(90)90033-9

[14] Tamura, T. and Miyagi, T. (1999) The Effect of Turbulence on Aerodynamic Forces on a Square Cylinder with Various Corner Shapes. Journal of Wind Engineering and Industrial Aerodynamics, 83, 135-145. http://dx.doi.org/10.1016/S0167-6105(99)00067-7

[15] Takizawa, N., Okada, N. and Iwasaki, A. (1985) Wind-Tunnel Investigation of the Pressure Distribution and Deduced 
Characteristics of Semi-Circular Cylinder in the Vicinity of Critical Reynolds Number. Technical Report of National Aerospace Laboratory, 871, 1-44. (In Japanese)

[16] Fujita, H. (2010) The Characteristics of the Aeolian Tone Radiated from Two-Dimensional Cylinders. Fluid Dynamics Research, 42, Article ID: 015002, 25 p.

[17] Fujisawa, N. (1992) On the Torque Mechanism of Savonius Rotors. Journal of Wind Engineering and Industrial Aerodynamics, 40, 277-292. http://dx.doi.org/10.1016/0167-6105(92)90380-S

[18] Stäger, R. and Echelmann, H. (1991) The Effect of Endplates on the Shedding Frequency of Circular Cylinders in the Irregular Range. Physics of Fluids A, 3, 2116-2121. http://dx.doi.org/10.1063/1.857894

[19] Curle, N. (1955) The Influence of Solid Boundaries upon Aerodynamic Sound. Proceedings of the Royal Society of London Series A, 231, 505-514. http://dx.doi.org/10.1098/rspa.1955.0191

\section{Nomenclature}

$C_{\mathrm{d}}, C_{\mathrm{l}}$ : Drag and lift coefficient, respectively

$C_{\text {lrms: }}$ : Fluctuating lift coefficient

$D$ : Diameter of cylinder

$F_{\mathrm{d}}, F_{\mathrm{l}}$ : Streamwise and normal fluid forces, respectively

Re: Reynolds number $(=U D / v)$

SPL: Sound pressure level $(\mathrm{dB})$

St: Strouhal number $(=f D / U)$

$U$ : Free stream velocity

$\theta$ : Angle of attack (see Figure 2)

$x, y$ : Streamwise and normal coordinates (see Figure 2) 